\title{
Preventive effect of erythromycin on experimental bleomycin-induced acute lung injury in rats
}

\author{
Arata Azuma, Tomoyuki Furuta, Tatsuji Enomoto, Yasushi Hashimoto, \\ Kazutsugu Uematsu, Naotaka Nukariya, Akira Murata, Shoji Kudoh
}

\begin{abstract}
Background-Erythromycin has been reported to have an inhibitory effect on chronic inflammatory airway disease and chronic infiltration of neutrophils into the airway. Bleomycin (BLM) often induces interstitial lung fibrosis following acute lung injury. A study was undertaken to investigate the effects of erythromycin (EM) on experimental bleomycin-induced acute lung injury in rats.

Methods-Bleomycin-induced lung injury was assessed by light microscopic examination, measurement of neutrophil elastase activity and of the interleukin 8 (IL-8) content in bronchoalveolar lavage (BAL) fluid. The potential inhibitory effect of erythromycin was assessed by overall comparison of erythromycin untreated (BLM alone), concurrently treated (BLM + EM), and pretreated (BLM + pre-EM) groups.

Results-The neutrophil count and concentration of neutrophil-derived elastase in BAL fluid were significantly different in the three groups. The morphological changes of lung injury were also less extensive in rats pretreated with erythromycin. However, these protective effects were not marked in the group concurrently treated with erythromycin. Moreover, the concentration of IL-8 in the BAL fluid tended to be less in the erythromycin treated groups; however, there were no significant differences between the bleomycin-treated groups.

Conclusion-Erythromycin exhibits a prophylactic effect on acute lung injury induced by intratracheal administration of bleomycin, which is possibly associated with a downregulation of neutrophilderived elastase.

(Thorax 1998;53:186-189)
\end{abstract}

Keywords: erythromycin; bleomycin; acute lung injury

Japan

A Azuma

T Furuta

T Enomoto

Y Hashimoto

K Uematsu

N Nukariya

A Murata

S Kudoh

Correspondence to: Dr A Azuma.

Received 14 March 1997 Returned to authors 14 April 1997

Revised version received

18 November 1997

Accepted for publication

21 November 1997 is indicated by a prominent accumulation of neutrophils in the lung parenchyma. ${ }^{45}$ These neutrophils cause progressive airway damage by the release of oxygen radicals and proteolytic enzymes, including neutrophil elastase. ${ }^{5-8}$ Interleukin-8 (IL-8) is a powerful cytokine inducing neutrophil accumulation into inflammatory sites. ${ }^{9-13}$
Diffuse panbronchiolitis (DPB) is a well known inflammatory airway disease in Mongolian nations ${ }^{14}{ }^{15}$; however, the prognosis for DPB has been improved significantly by long term, low dose erythromycin treatment. ${ }^{16}{ }^{17}$ In patients receiving oral erythromycin the increase in neutrophils in bronchoalveolar lavage (BAL) fluid was significantly reduced. ${ }^{18}$ In one study in which a lipopolysaccharide-induced acute lung injury in a rat model was used to study the effect of erythromycin it was found that erythromycin inhibited the release of IL-8 into the airways. ${ }^{19-21}$ While consensus regarding the primary mechanism by which erythromycin improves the prognosis of DPB has not been established, it is possibly the result of its known anti-inflammatory effect. ${ }^{22}{ }^{23}$

This study examined the inhibitory effects of erythromycin in bleomycin-induced lung injury and evaluated the mechanisms of the prophylactic effect of erythromycin on acute lung oedema and neutrophil chemotaxis in rats.

\section{Methods}

Male, four week old, Sprague-Dawley rats weighing an average of $250 \mathrm{~g}$ each were randomly divided into treatment groups for administration of the following agents: bleomycin (BLM) (Nippon Kayaku, Co Ltd, Tokyo, Japan) at $4.5 \mathrm{mg} / \mathrm{kg}$ in normal saline $(0.6 \mathrm{ml})$ instilled into the trachea under general anaesthesia by ether at day 14, and/or erythromycin (EM) (Dainabott, Co Ltd, Osaka, Japan) $12 \mathrm{mg} / \mathrm{kg}$ in $10 \%$ ethanol administrated orally for seven or 21 days.

The number of rats ranged from 13 to 20 per group, and groups were defined as follows: group 1: BLM alone ( $\mathrm{n}=15)$; group 2: $\mathrm{BLM}+$ EM (from day 14 to day $21, \mathrm{n}=15$ ); group 3 : $\mathrm{BLM}+$ pre-EM (from day 1 to day $21, \mathrm{n}=20$ ); group 4: EM alone (from day 1 to day 21, $\mathrm{n}=$ 13); and saline control (instilled into the trachea at day 14).

All rats were sacrificed at day 21 for evaluation of lung pathology, BAL cells and fluid. In the EM alone and $10 \%$ ethanol/saline groups, no remarkable findings were found. However, one rat in the BLM alone group, two in the $\mathrm{BLM}+\mathrm{EM}$ group, and four in the BLM + pre-EM group died from acute aspiration pneumonia (fig 1).

Rats were sacrificed under ether anaesthesia at day 7 after BLM treatment. BAL was performed by injection of $5 \mathrm{ml}$ saline each $(25 \mathrm{ml}$ total) into the trachea through an intratracheal tube. ${ }^{24}$ Recovery ratios of lavage fluids ranged 

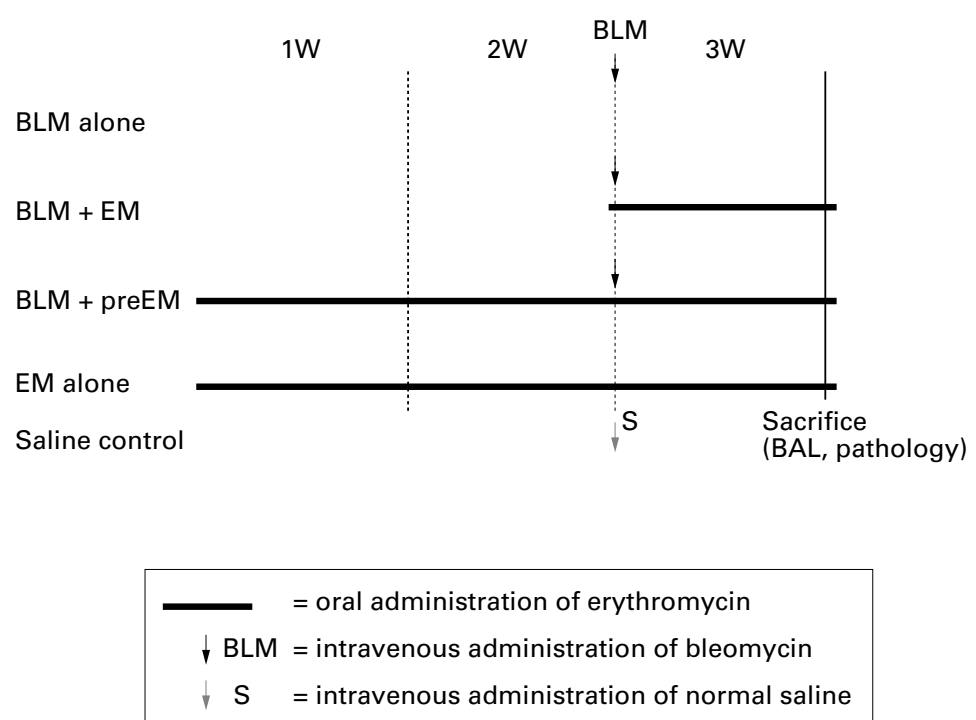

Figure 1 Bleomycin (BLM) and erythromycin (EM) administration schedules for each group.

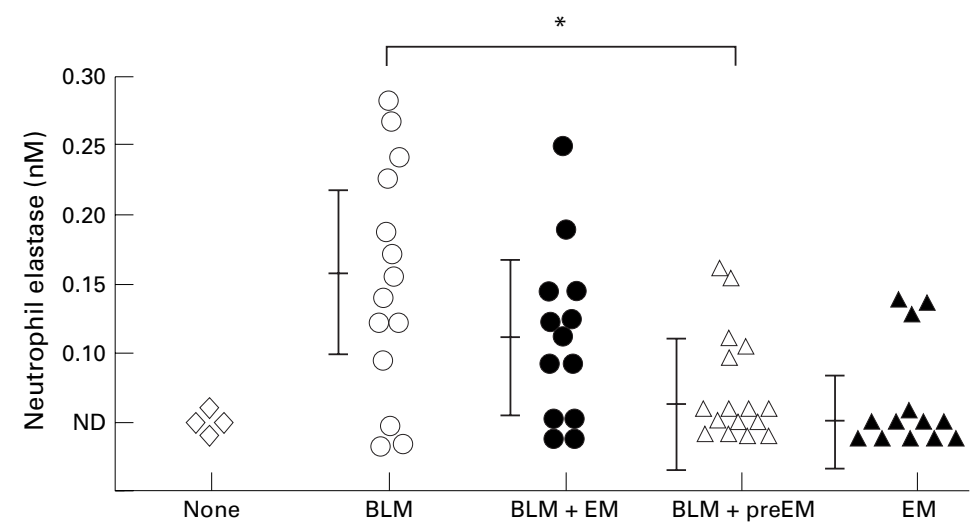

Figure 2 Comparison of neutrophil elastase activity in BAL fluid between groups. The activity of the BLM + pre-EM group was significantly inhibited compared with that of the $B L M$ alone group $\left({ }^{*} p<0.10\right) . N D=$ not detectable $(<0.10 \mathrm{nM})$.

from $60 \%$ to $70 \%$ and were not significantly different among groups. BAL cells were separated by centrifugation at $1500 \mathrm{rpm}$ for 10 minutes and were used for measurement of neutrophil elastase activity, IL-8 content, cell population, and cell number.

Neutrophil elastase

Neutrophil elastase activity in BAL fluid was measured with a photoreader by measuring the change in substrate methoxy-succinyl-alanylalanyl-prolyl-valyl $p$-nitroanilide (Sigma, USA). ${ }^{25-27}$

Table 1 Comparison of total cell count in BAL fluid, percentage of neutrophils, and neutrophil counts among four groups

\begin{tabular}{|c|c|c|c|c|c|c|}
\hline \multirow{2}{*}{$\frac{\text { Group }}{\text { BLM alone }}$} & \multicolumn{2}{|c|}{$\begin{array}{l}\text { No. of } B A L \\
\text { cells }\left(\times 10^{3} / \mathrm{ml}\right)\end{array}$} & \multicolumn{2}{|l|}{$\%$ Neutrophils } & \multicolumn{2}{|c|}{$\begin{array}{l}\text { Neutrophil } \\
\text { counts }\left(\times 10^{3} / \mathrm{ml}\right)\end{array}$} \\
\hline & $334(155)$ & & $45.6(17.1)$ & & $156.9(26.5)$ & \\
\hline $\mathrm{BLM}+\mathrm{EM}$ & $201(76)$ & $\star$ & $34.7(21.7)$ & $\star$ & $69.7(16.5)$ & \\
\hline $\mathrm{BLM}+$ pre-EM & $194(49)$ & & $8.6(6.9)$ & & $17.0(3.4)$ & \\
\hline EM alone & $148(56)$ & & ND & & $\mathrm{ND}$ & \\
\hline Saline & $150(45)$ & & ND & & ND & \\
\hline None & $135(30)$ & & ND & & ND & \\
\hline
\end{tabular}

${ }^{\star} \mathrm{p}<0.01$ difference between these three groups.

$\mathrm{ND}=$ not detectable; $\mathrm{BLM}=$ bleomycin; $\mathrm{EM}=$ erythromycin.
IL-8 assay

IL-8 concentration in BAL fluid was measured with an enzyme-linked immunosorbent assay (ELISA) kit (Panafarm Lab, Kumamoto, Japan). ${ }^{28}$

Biopsy, histology, and graphical estimation

Lung tissue was removed and stained with haematoxylin-eosin (HE) for light microscopic evaluation. "Interstitial oedema" and "infiltration of inflammatory cells" were photographically evaluated as a bleomycin-associated acute lung injury. Adobe Photoshop 3.0J was used to detect these areas, and five longitudinal lung sections in each group were used for evaluation. The "ratio" was indicated by the number of black pixel areas.

\section{STATISTICAL ANALYSIS}

The statistical significance among the three groups of animals was tested with the KruskalWallis rank test, a $\mathrm{p}$ value of less than 0.01 being considered significant. If significant, partwise comparisons between the two groups were performed using the Mann Whitney nonparametric test, a $\mathrm{p}$ value of less than 0.01 again being considered significant.

\section{Results}

BAL FLUID CELL COUNT

The mean (SD) total BAL fluid cell count in rats treated with BLM alone was $344(155) \times$ $10^{3} / \mathrm{ml}$ ) compared with 201 (76) for BLM + EM, 194 (49) for BLM + pre-EM, and 148 (56) for EM alone. Differences between the three groups (BLM alone, BLM + EM, and $\mathrm{BLM}+$ pre-EM) were statistically significant (table 1).

NEUTROPHIL RATIO IN BAL CELLS

The mean (SD) neutrophil ratios were 45.6 $(17.1) \%$ in the BLM alone group, 34.7 (21.7)\% in the BLM + EM group, $8.6(6.9) \%$ in the BLM + pre-EM group, and $0.0 \%$ in the EM alone group. Significant differences were revealed in all of the groups. The mean (SD) neutrophil count was calculated as 156.9 $(26.5) \times 10^{3} / \mathrm{ml}$ for BLM alone, $69.7(16.5) \times$ $10^{3}$ for BLM + EM, 17.0 (3.4) $\times 10^{3}$ for BLM + pre-EM, and 0 (0) for EM alone (table 1).

NEUTROPHIL ELASTASE ACTIVITY IN BAL FLUID The sensitivity of the assay for neutrophil elastase was $0.1 \mathrm{nM}(\mathrm{nmol} / \mathrm{l})$. For BLM alone, 11 of 14 cases $(71.4 \%)$ were measurable; for $\mathrm{BLM}+\mathrm{EM}$, nine of 13 cases $(69.2 \%)$; for BLM + pre-EM, five of 16 cases $(31.3 \%)$; and for EM alone, three of 13 cases (23.0\%). There were significant differences between the three groups (BLM alone, BLM + EM, and BLM + pre-EM) and Mann Whitney's U test revealed statistical significance between BLM alone and $\mathrm{BLM}+$ pre-EM $(\mathrm{p}<0.01)($ fig 2$)$.

HISTOLOGICAL ASSESSMENT OF ACUTE LUNG INJURY

Areas of cell infiltration and oedema were taken as an indication of acute lung injury and were assessed by graphical software Photoshop 3.0 J and stained with black colour. The ratio 


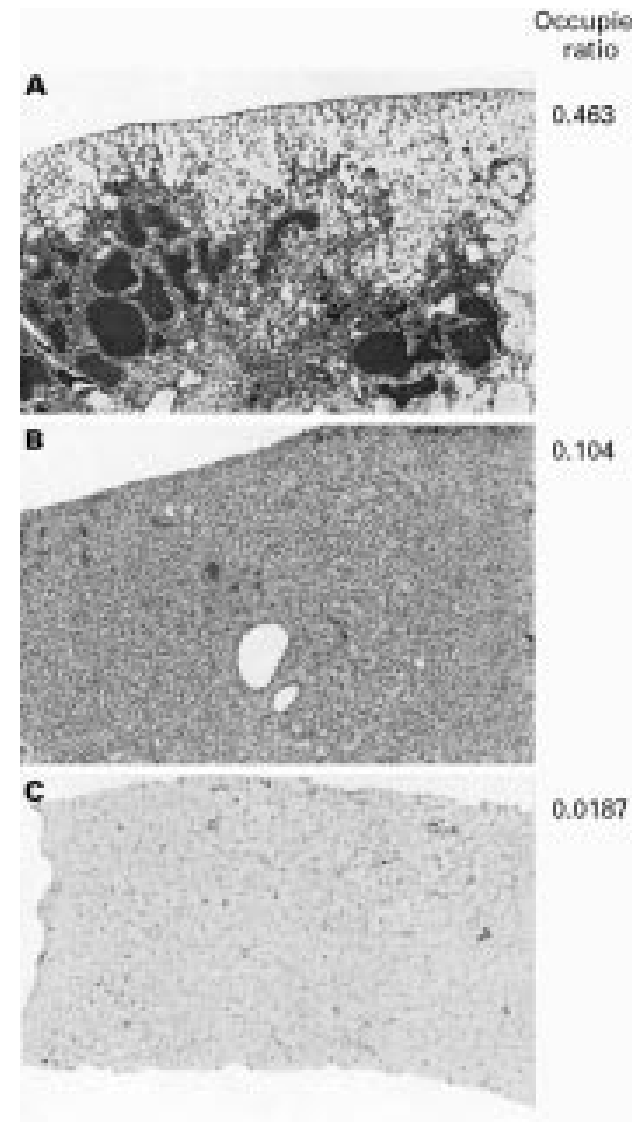

Figure 3 Typical views of pathology graphically modified by Photoshop 3.07 graphic software in (A) the BLM alone group, $(B)$ the $B L M+$ pre-EM group, and $(C)$ the normal saline group. The number of pixels in the area stained black was counted as acute lung injury. The ratio was 0.463 in the BLM alone group, 0.104 in the BLM + pre-EM group, and 0.0187 in the normal saline group. These ratios were decreased in the erythromycin pretreated group.

was 0.463 in the BLM alone group, 0.104 in the BLM + pre-EM group, and 0.0187 in the EM alone group (fig 3).

IL-8 CONTENT IN BAL FLUID

The concentration of IL-8 in the BAL fluid at day 7 after BLM inoculation was 0.692 $(0.265) \mathrm{ng} / \mathrm{ml}$ in the BLM alone group, 0.429 (0.249) $\mathrm{ng} / \mathrm{ml}$ in the BLM + EM group, 0.452 $(0.305) \mathrm{ng} / \mathrm{ml}$ in the BLM + pre-EM group, and $0.389(0.149) \mathrm{ng} / \mathrm{ml}$ in the EM alone group, which were not significantly different (fig 4).

NS

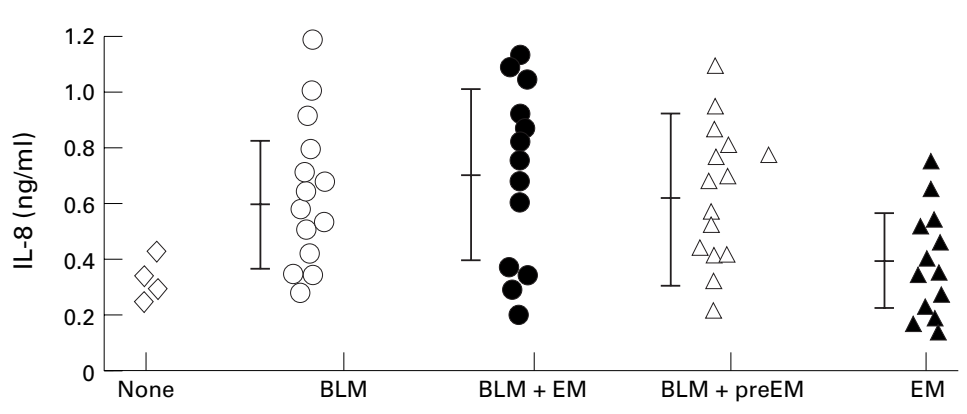

Figure 4 Median (SD) levels of IL-8 in BAL fluid measured by the ELISA method. The levels were not significantly different (NS) in any of the groups but tended to decrease in the BLM + pre-EM group compared with the BLM alone group.

\section{Discussion}

This study analysed the inhibitory effect of erythromycin on bleomycin-induced acute lung injury and found that, by pathological analysis, the most marked changes such as interstitial oedema and infiltration of inflammatory cells into the lung were induced at day 7 following bleomycin treatment.

Pretreatment with erythromycin prior to bleomycin + erythromycin decreased the 2.29fold increase in total BAL cell count with bleomycin alone to a 1.32-fold increase compared with that with erythromycin alone. The neutrophil ratio in the BAL fluid in the BLM + EM group was $0.444(69.7 / 156.9)$ compared with that of the bleomycin alone group. Furthermore, the neutrophil ratio in the BLM + pre-EM group was 0.106 , which indicated that erythromycin caused marked inhibition of neutrophil infiltration into the airway in bleomycin-induced lung injury. An inhibitory effect of erythromycin on neutrophil chemotaxis in patients with chronic bronchitis and diffuse panbronchiolitis has been reported previously. ${ }^{10} 1618$ This study suggests a similar mechanism of action for erythromycin since inhibition to neutrophil chemotaxis was related to bleomycin-induced acute lung injury (table $1)$.

The effect of erythromycin on neutrophil elastase activity was also examined. Neutrophil elastase, in general, increases vascular leakage, ${ }^{19}{ }^{20}$ induces neutrophil chemotaxis in tissues, ${ }^{7}$ and injures bronchial epithelial cells. ${ }^{89}$ In the present study erythromycin significantly inhibited neutrophil elastase activity in the BAL fluid of the BLM + pre-EM group compared with the BLM alone group (fig 2). However, in the BLM + EM group the inhibitory effect of erythromycin on neutrophil elastase activity was not significant despite a $50 \%$ reduction of neutrophils in the airway (fig 2 , table 1). These findings suggest that erythromycin may have inhibited neutrophil chemotaxis into the airway but did not directly inactivate neutrophil elastase activity in BAL fluid, similar to the erythromycin-induced improvement in chronic bronchial inflammatory disease previously reported. ${ }^{10}$ Erythromycin has been reported to inhibit the release of oxygen radicals from neutrophils, which contributes to the prevention of lung injury. ${ }^{91920}$ In view of Tamaoki's report, increased cAMP may also be involved in preventing vascular leakage. ${ }^{29}$

IL-8, cloned by Matsushima, ${ }^{11}$ is known to promote neutrophil chemotaxis and may be present in the progressive phase of bleomycininduced lung injury and fibrosis. In the present study the average IL-8 concentration in the BAL fluid was $0.389 \mathrm{ng} / \mathrm{ml}$ in the group given normal saline and $0.692 \mathrm{ng} / \mathrm{ml}$ in the bleomycin alone group, which was not significantly different. There were no significant differences between the four groups. Since IL- 8 can be released from multiple cell sources (epithelial cells, vascular endothelial cells, interstitial cells and inflammatory cells ${ }^{12}{ }^{13}$ ), the distribution of IL-8 in the lung tissue may be complicated. Analysis of the inhibitory effect of IL- 8 by the 
anti-IL-8 antibody will be useful for evaluating the role of IL-8 in bleomycin-induced lung injury. The inhibitory effect of erythromycin on IL-8 production has been confirmed. ${ }^{21}$ Further studies are needed to elucidate the precise effect of erythromycin on IL-8, whether direct or through other cytokines such as TNF- $\alpha$ or IL- $1 \beta .{ }^{11}$

In summary, erythromycin inhibited neutrophil infiltration into the airway of rats included in this study. Neutrophil elastase activity was accompanied by inhibition of the neutrophil count, and bleomycin-induced acute lung injury was associated with neutrophil elastase activity. Prior treatment with erythromycin was more effective on bleomycin-induced acute lung injury than simultaneous treatment with erythromycin; however, IL-8 as a strong neutrophil chemotactic factor tended to be reduced by erythromycin but was not significantly different when compared with the bleomycin alone group. Significant inhibition of neutrophil numbers and neutrophil elastase activities in BAL fluid by erythromycin appeared to be less related to the IL- 8 content of the BAL fluid.

1 Anderson IYR, Bowden DH. The pathogenesis of bleomycin-induced pulmonary fibrosis in mice. Am $\mathcal{F}$ Pathol 1974;77:185-98.

2 Aso Y, Yoneda K, Kikkawa Y. Morphologic and biochemical study of pulmonary changes induced by bleomycin in mice. Lab Invest 1976; 35:558-68.

3 Weidner WJ, Quam DA, McClure DE, et al. Effect of acute administration of bleomycin on lung fluid balance in sheep. Exp Lung Res 1995;21:617-30.

4 Mitsuhashi H, Asano S, Nonaka T, et al. Administration of truncated secretory leukoprotease inhibitor ameliorates
bleomycin-induced pulmonary fibrosis in hamsters. $A m \mathcal{F}$ Respir Crit Care Med 1996;153:369-74.

5 Nagai A, Aoshiba K, Ishihara Y, et al. Administration of alpha 1-proteinase inhibitor ameliorates bleomycinCare Med 1992;145:651-6.

6 Melby K, Towes GB, Pierce AK. Pulmonary elastase activity in response to Streptococcus pneumoniae and Pseudomonas aeruginosa. Am Rev Respir Dis 1985;131:559-63.

7 Persson CG, Erjefalt I. Inflammatory leakage of macromolecules from the vascular compartment into the tracheal ecules from the vascular compartment in
lumen. Acta Physiol Scand 1986;126:615-6.

8 Tegner H, Ohlsson K, Toremalm NG, et al. Effect of human leucocyte enzymes on tracheal mucosa and its mucociliary leucocyte enzymes on tracheal muco
activity. Rhinology 1979;17:199-206.

9 Janoff A, Sloan B, Weinbaum G, et al. Experimental emphysema induced with purified human neutrophil elastase: tissue localization of the instilled protease. Am Rev Respir Dis 1977;15:461-78.
10 Mikami M. Clinical and pathophysiological significance of neutrophil elastase in sputum and the effect of erythromycin in chronic respiratory diseases. Fap $\mathcal{F}$ Thorac Dis 1991;29:72-83

11 Matsushima K, Morishita K, Yoshimura T, et al. Molecular cloning of a human monocyte-derived neutrophil chemotactic factor (MDNCF) and the induction of the MDNCF mRNA by interleukin 1 and tumor necrosis factor. $\mathcal{F}$ Exp Med 1988;167:1883-93.

12 Strieter RM, Phan SH, Showell HJ, et al. Monokine-induced neutrophil chemotactic factor gene expression in human fibroblasts. 7 Biol Chem 1989;264:10621-6.

13 Schroder JM, Morwietz U, Morita E, et al. Purification and partial biochemical characterization of a human monocytederived, neutrophil-activating peptide that lacks interleukin 1 activity. F Immunol 1987;139:3474-88.

14 Homma H. Definition of diffuse panbronchiolitis. $\mathcal{F}$ fpn Soc Intern Med 1976;65:649-59.

15 Homma H, Yamanaka A, Tanimoto H, et al. Diffuse panbronchiolitis: a disease of the transitional zone of the lung. Chest 1983;83:63-9.

16 Kudoh S, Uetake T, Hagiwara K, et al. Clinical effect of low dose long term erythromycin chemotherapy on diffuse panbronchiolitis. fap 7 Thorac Dis 1987;25:632-42.

17 Kadota J, Sakito O, Kohno S, et al. A mechanism of erythromycin treatment in patients with diffuse panbronchiolitis. Am Rev Respir Dis 1993;147:153-9.

18 Oda H, Kadota J, Kohno S, et al. Erythromycin inhibits neutrophil chemotaxis in bronchoalveoli of diffuse panbronchiolitis. Chest 1994;106:1116-23.

19 Tamaoki J, Sakai N, Tagaya E, et al. Macrolide antibiotics protect against endotoxin-induced vascular leakage and neutrophil accumulation in rat trachea. Antimicrob Agents Chemother 1994;38:1641-3.

20 Tamaoki J, Tagaya E, Yamawaki I, et al. Effect of erythromycin on endotoxin-induced microvascular leakage in the rat trachea and lungs. Am f Respir Crit Care Med 1995;151: 1582-8.

21 Oishi K, Sonoda F, Kobayashi S, et al. Role of interleukin-8 (IL-8) and an inhibitory effect of erythromycin on IL-8 release in the airways of patients with chronic airway diseases. Infect Immun 1994;62:4145-52.

22 Eyraud A, Descotes J, Lombard JY, et al. Effects of erythromycin, josamycin and spiramycin on rat polymorphonuclear leukocyte chemotaxis. Chemotherapy 1986;32:379-82.

23 Nelson S, Summer WR, Terry PB, et al. Erythromycininduced suppression of pulmonary antibacterial defenses: a potential mechanism of superinfection in the lung. Am Rev Respir Dis 1987;136:1207-12.

24 Nelson S, Summer WR, Bagby G, et al. Granulocyte colony-stimulating factor enhances pulmonary host defenses in normal and ethanol-treated rats. F Infect Dis 1991; 164:901-6.

25 Twumasi DY, Liener IE. Proteases from purulent sputum. Purification and properties of the elastase and chymotrypsin-like enzymes. F Biol Chem 1977;252:191726.

26 Ohlsson K, Tegner H. Granulocyte collagenase, elastase and plasma protease inhibitors in purulent sputum. Eur $\mathcal{F}$ Clin Invest 1975;5:221-7.

27 Toews GB, Vial WC. The role of C5 in polymorphonuclear leukocyte recruitment in response to Streptococcus pneumoniae. Am Rev Respir Dis 1984;129:82-6.

28 Murata Y, Ishiguro Y, Itoh J, et al. The role of proinflammatory and immunoregulatory cytokines in the pathogenesis of ulcerative colitis. F Gastroenterol 1995;30(Suppl):56-60.

29 Tkeyama K, Tamaoki J, Chiyotani A, et al. Effect of Tkeyama K, Tamaoki J, Chiyotani A, et al. Effect of
macrolide antibiotics on ciliary motility in rabbit airway epithelium. F Pharm Phamacol 1993;45:756-8. 